The role of working memory capacity in evaluative judgments of liking and beauty

Jiajia Che ${ }^{1}$, Xiaolei Sun ${ }^{1}$, Martin Skov ${ }^{2,3}$, Oshin Vartanian ${ }^{4}$, Jaume Rosselló ${ }^{1}, \&$ Marcos Nadal $^{1}$

1 Department of Psychology, University of the Balearic Islands, Spain

2 Decision Neuroscience Research Cluster, Copenhagen Business School, Denmark

2 Danish Research Centre for Magnetic Resonance, Copenhagen University Hospital Hvidovre, Denmark

4 Department of Psychology, University of Toronto, Canada

Address for correspondence:

Marcos Nadal. Department of Psychology. University of the Balearic Islands. Crta Valldemossa Km 7.5 Palma de Mallorca 07122 Spain. Email: marcos.nadal@uib.es

Data availability: The data that support the findings of this study are openly available in Open Science Framework at https://osf.io/5xw3v/?view_only=bc77e10ed55147ab82612f96a55c5b78

Keywords: aesthetic judgment, working memory, working memory capacity, hedonic valuation, liking, beauty 


\title{
The role of working memory capacity in evaluative judgments of liking and beauty
}

\begin{abstract}
Judgments of liking and beauty appear to be expressions of a common hedonic state, but they differ in how they engage cognitive processes. We hypothesized that beauty judgments place greater demands on limited executive resources than judgments of liking. We tested this hypothesis by asking two groups of participants to judge works of visual art for their beauty or liking while having to remember the location of 1,3 , or 5 dots in a 4 by 4 matrix. We also examined the effect of individual differences in working memory capacity. Our results show that holding information about the location of the dots in working memory delayed judgments of beauty but not of liking. Also, the greater participants' working memory capacity, the faster they completed the working memory task when judging liking, but not when judging beauty. Our study provides evidence that judging beauty draws more on working memory resources than judging liking.
\end{abstract}

Keywords: aesthetic judgment, working memory, working memory capacity, beauty, liking. 


\section{The role of working memory capacity in evaluative judgments of liking and beauty}

Hedonic valuation is a fundamental feature of human cognition. We continually value the current or future pleasurableness of objects, situations, events, or courses of action in the light of present cognitive, affective, and physiological states and goals. This value is crucial for making decisions, comparing and choosing among alternatives, and prioritizing actions (Berridge \& Kringelbach, 2015). Among the least understood forms of hedonic valuation are evaluative judgments: assessments of how good, beautiful, or pleasant objects are, how much we like them, or which we prefer (Berlyne, 1973).

Different types of evaluative judgments, such as pleasingness, liking, beauty, or pleasure are grounded on a common hedonic tone, but they are not equivalent. For instance, Russell and George (1990) found that painting style influenced pleasing judgments, but not liking judgments, and Marin and colleagues (2016) found that complexity influences judgments of beauty, pleasantness and liking are influenced by in different ways (positive, negative, inverted-U), and depend on the kind of object being evaluated (images from the International Affective Picture System, cartoons, paintings, and music excerpts). Evaluative judgments, thus, differ from each other in how they engage perceptual and cognitive processes associated with value assessment. In sum, although different evaluative judgments seem to stem from a common hedonic value, they appear to engage cognitive processes in different ways (Leder et al., 2005).

There is evidence to suggest that some of the differences between evaluative judgments owe to differences in the way they engage working memory (WM). Sherman and colleagues (2015) found that aesthetic appreciation was greater when the degree of visual complexity was compatible with the observer's visual WMC: Participants with greater visual WMC reported 
higher ratings for complex paintings. Brielmann and Pelli (2017) asked participants to rate how much pleasure they felt when viewing images that had been classified as beautiful or not beautiful, and whether they found them beautiful or not. This aesthetic rating occurred either in conjunction or not with an auditory n-back task that increased WM load. The n-back task reduced beauty and pleasure ratings for beautiful stimuli but had no effect on the beauty ratings for non-beautiful stimuli. Mullennix and colleagues (2015) investigated how manipulating memory engagement affects liking judgments. They instructed participants to rate photographs on liking while performing a concurrent digit preload memory recall task (i.e., remembering a string of letters). The results showed that liking was unaffected by increases in digit load. Mullennix et al. (2018) used abstract modern artworks and a concurrent digit preload memory recall task. They found that higher memory load unexpectedly increased liking for abstract artworks. The reason for this unexpected effect was unclear, although the authors conjectured that increased attentional focus on the memory task in the high-load condition may have "leaked over" to the artworks.

These studies suggest that hedonic valuation relies on WMC, and that WM processes seem to be engaged differently by beauty and liking judgments. WM is a storage and processing cognitive system that enables actively keeping information required for ongoing processes in an accessible state (Cowan, 2017; Shipstead, Harrison, \& Engle, 2016). Working memory capacity (WMC) is a measure of individual differences in the efficacy of this system's function (Shipstead, Harrison, \& Engle, 2015). The executive attention account of WMC (Engle, 2018) considers that differences in WMC owe to differences in the ability to control attention with the aim of maintaining access to goal-relevant information, rather than responding reflexively (Shipstead et al., 2015). It is possible, therefore, that whereas liking judgments reflect primarily 
hedonic signals, beauty judgments require integrating those signals with the comparison of the perceptual features of an object with learnt beauty templates. This additional requirement would involve holding perceptual information online while template-matching processes are carried out, probably taking a longer time.

In sum, evaluative judgments stem from a common hedonic tone, but seem to make different demands on working memory resources. Brielmann and Pelli's (2017) results suggest that working memory is essential in the computation of beauty judgments. Mullennix and colleagues' $(2015,2018)$ results suggest that working memory is not essential in the computation of liking judgments. To resolve this apparent discrepancy, and to clarify whether liking and beauty judgments differ in the way in which they engage WM processes, we designed an experiment where participants judged liking or beauty for paintings while their WM was loaded by a concurrent visuospatial WM task. We measured participants' visuospatial WMC to determine its influence on responses and response times. Given that we presented participants with two concurrent tasks that draw on WM resources (WM task and evaluative judgment task), we expected performance, measured in terms of the outcome of the processing (responses) and in terms of the time of processing (response times), to decrease with increasing difficulty. Thus, we expected to find evidence for the differential engagement of WM in beauty and liking evaluative judgments in responses and response times. We also expected a mutual influence between the concurrent WM and judgment tasks: we expected to find evidence for differences in how judgments of liking and beauty engage WM resources in the influence of WM manipulations on participants' responses and response times in the evaluative judgment task, and also in the influence of the evaluative judgment task participants had been asked to perform (either beauty or liking) on performance in the WM task. 
Given the small number of studies on the role of executive functions in evaluative judgments, and given that this is the first study to directly compare the role of WM in beauty and liking judgments, our hypotheses should be understood as exploratory. Based on the literature reviewed above, we expected beauty judgments to make greater demands on WM resources than liking judgments. If this were the case, we would find, on the one hand, lower accuracy and slower response times in the WM task when participants judged beauty than when they judged liking, especially when WM load was intermediate and high. On the other hand, we would find changes in judgments and slower judgment times as the load of the WM task increased, especially when judging beauty. We also expected WMC to moderate these effects, with participants with greater WMC less susceptible to the manipulations than participants with lower WMC.

\section{Method}

\section{Participants}

One hundred and thirty-seven students ( 90 women, mean age=21.97, $S D=5.51$ ) at the University of the Balearic Islands with normal or corrected-to-normal vision volunteered for the experiment. Participants were fully briefed about the procedure and signed an informed consent before testing.

\section{Materials and procedure}

\section{Aesthetic Judgment and WM Concurrent Tasks}

Participants performed two concurrent tasks: judging how much they liked or how beautiful they found a series of paintings (aesthetic judgment task) while having to remember the 
location of 1, 3, or 5 dots in a 4 by 4 grid (WM task). In the WM task, participants viewed and had to remember dot-in-matrix patterns (Ichikawa, 1983): 16 contained one dot (low WM load condition), 16 contained three dots (medium WM load condition), and 16 contained five dots (high WM load condition). Participants were randomly assigned to two judgment groups: 70 were asked to judge beauty, and 67 were asked to judge liking. Participants in both groups performed the judgment task using a 5-point Likert scale with the following anchors: Do not like at all - Like very much; Not at all beautiful - Very beautiful.

The set of artworks included 48 western artistic paintings depicting rural or urban landscapes. Each participant rated all the paintings, but under different WM load condition: all participants judged 16 artworks in the low WM condition, 16 in the medium WM condition, and 16 in the high WM condition, just not the same artworks. WM load conditions were presented in blocks, that is to say, participants completed 16 consecutive trials under the same WM load. The order of the WM load blocks was randomized for each participant, and so was the order of the artworks and of the dot-in-matrix figure within each block.

Trials began with a dot-in-matrix presented for 750ms (Miyake et al., 2001). Participants had to remember the layout of the dots. After the offset of the dot-in-matrix figure, one of the artistic paintings was shown for $2,000 \mathrm{~ms}$, after which a response was prompted by showing the corresponding judgment task ("How much do you like this image" or "How beautiful do you find this image?"). Participants used the mouse to click on the number corresponding to their rating. After this, an empty 4 by 4 grid appeared, which prompted participants to click in the cells that contained the dots in the same arrangement as they had seen at the start of the trial. After their response, a new trial began. Before the test, participants were given six practice trials. We set no time limit for responses in the judgment task or in the WM task. 


\section{WMC Task}

We used an operation span task that required participants to verify a matrix equation while remembering the location of a dot in a 5 by 5 grid. The basic unit of each trial was a matrix addition or subtraction equation that needed verifying followed by a 5 by 5 grid containing one dot. Participants had a maximum of 4.5 seconds to verify each equation by using the mouse to click on the "True" or "False" icons, and the dot grid was displayed for $1500 \mathrm{~ms}$ (Miyake et al., 2001).

After a sequence of between two and five equation-grid units (Conway et al., 2005), participants had to recall the positions of the dots in the same sequence as they had appeared throughout the sequence. The sets increased in size from two to five equations and dots ( 4 of each) for a total of 16 sets (Miyake et al., 2001). To ensure that participants attended to the equation verification, the instructions emphasized that they should make sure to verify the equations correctly (Conway et al., 2005). Partial-credit unit scoring was used to calculate WMC for each participant (Friedman \& Miyake, 2005; Conway et al., 2005). There were three practice trials with two equations and two dots each.

\section{Data Analysis}

Participants' responses in the aesthetic judgment and WM tasks were analyzed using linear mixed effects models (Judd et al., 2017). Linear mixed effects models account simultaneously for the between-subjects and within-subject effects of the independent variables (Baayen et al., 2008), unlike ANOVAs. ANOVAs usually require averaging across stimuli, which can cause the empirical Type-I error rate to greatly exceed the nominal level, and lead to 
claims of significant effects that are unlikely to replicate with different samples (Judd et al., 2017).

The models were set up to reflect the effect of task (beauty or liking judgment), memory load (low, medium, high), and visuospatial WMC, on participants' responses: Whether they produced the correct matrix in the WM task, and the rating given in the beauty or liking task. We modelled the maximal random effects structure. This avoids the loss of power, reduces Type-I error, and enables the generalizability of results to other participants and stimuli (Barr et al., 2013): The models of aesthetic judgments (outcome 1 to 5) and WM task (outcome correct / incorrect matrix) included the interaction between rating task (beauty, liking), memory load (low, medium, high), and WMC (continuous) as fixed effects. They also included the intercept and slope for memory load as random effects within participants, and the intercept and slope for rating task as random effects within stimuli. The analyses were carried out in $\mathrm{R}$ (R Core Team, 2020), using the mixed() function of the 'afex' package (Singmann et al., 2015). Follow-up paired comparisons were conducted using the test () and emtrends $($ ) functions of the R package 'emmeans' (Lenth, 2018).

Response times in the WM and the aesthetic judgment tasks were subjected to exGaussian distribution analysis (Brewer, 2011). The ex-Gaussian is described by three parameters (Heathcote et al., 1991): $\mu$, the mean of the Gaussian component, $\sigma$, the standard deviation of the Gaussian component, and $\tau$, the mean and standard deviation of the exponential component. Before analyzing the RT data, we excluded anomalously short and long RTs (Baayen \& Milin, 2010): trials with RTs under $150 \mathrm{~ms}$ and over the $3^{\text {rd }}$ quartile +1.5 times the interquartile range. We then used the timefit() function of the 'retimes' package (Massidda, 2013) running in R ( $\mathrm{R}$ Core Team, 2020) to fit the ex-Gaussian function to each individual participant's RT data from 
each combination of judgment (beauty and liking) and memory load (high, medium, and low). After obtaining the three ex-Gaussian parameters for each participant in each condition, we used linear regression analyses to determine the effects of judgment (beauty, liking), memory load (low, medium, high), WMC (centered continuous variable), and their interactions on $\mu, \sigma$, and $\tau$.

\section{Results}

\section{Working Memory Capacity}

The mean WMC of the group of participants who rated the beauty of the artworks was 0.63 $(S D=0.14)$, and the mean WMC of the group of participants who rated how much they liked them was 0.63. $(S D=0.14)$. Conway and colleagues $(2005)$ recommended disregarding the WMC values for participants who make frequent mistakes in the processing component of the WMC task (in this case, the equation verification scores), which indicates questionable WMC scores. We therefore removed the WMC data for participants whose verification score was below 0.75 from the ensuing analyses (Conway et al., 2005): 28 participants (10 in the beauty rating group, 18 in the liking rating group). After this, the mean WMC of the group of participants who rated the beauty of the artworks was $0.65(S D=0.14)$, and the mean WMC of the group of participants who rated how much they liked them was 0.64. $(S D=0.14)$.

\section{Effects of Task, Memory Load, and WMC on Accuracy in the Memory Task}

We had hypothesized that, if beauty and liking judgments make different demands on WM resources, we would find an effect of judgment task on the accuracy in the memory task, mediated by WMC. WM load had a significant effect on the WM task performance: In the low load condition, participants reproduced more dot matrices correctly $(96.6 ; 95 \%$ CI $[95.5,97.5])$ 
than in the medium load condition $(71.7 \%[68.6,74.6] ; \beta=2.427, t=15.280, p<.0001$, HolmBonferroni corrected), and more in the medium load condition than in the high load condition (58.0\% [53.6, 62.2]; $\beta=0.607, t=6.214, p<.001$, Holm-Bonferroni corrected). The effect of WMC on performance was also significant: the greater the memory capacity, the better the performance on the WM task $(\beta=0.462, t=6.394, p<.001)$. There was a significant interaction between WMC and WM load $(\beta=-0.311, t=2.210, p=.0271)$ : The effect of WMC was stronger in the high WM load condition $(\beta=0.597[0.415,0.779])$ than in the low load condition $(\beta=0.239[-0.025,0.502])$, and stronger in the medium load condition $(\beta=0.550[0.399,0.701])$ than in the low load condition $(\beta=0.239[-0.025,0.502])$. No other main effect or interaction was significant (all $p s>.387$ ). In sum, participants made more mistakes in the visual WM task when they had to store more information within each trial, and made fewer mistakes if they had higher WMC, especially in the high and medium WM load conditions. These results serve as a manipulation check and validate the measures of WM load and WMC.

\section{Effects of Task, Memory Load, and WMC on Response Times in the Memory Task}

We had hypothesized that, if beauty and liking judgments make different demands on WM resources, we would find an effect of judgment task on response times in the memory task, mediated by WMC. We first excluded trials with RTs under $150 \mathrm{~ms}$ and over the 3rd quartile + 1.5 times the interquartile range (455 trials, $6.92 \%$ of the total). For the $\mu$ component of the exGaussian RT distribution the intercept was $\beta_{0}=2241.1 \mathrm{~ms}(t=91.718, p<.0001)$. The $\mu$ component was significantly greater in the high WM load condition $(3507 \mathrm{~ms}, 95 \%$ CI [3425, $3590])$ than in the medium WM load condition $(2437 \mathrm{~ms}[2354,2520] ; \beta=1070.4, t=17.885, p$ $<.0001$, Holm-Bonferroni corrected), and significantly greater in the medium WM load 
condition than in the low WM load condition (779 ms [696, 862]; $\beta=1657.8, t=27.669, p<$ .0001, Holm-Bonferroni corrected). WMC also had a significant effect on the $\mu$ component: The greater participants' WMC, the smaller the $\mu$ component of their RT distributions ( $\beta=-57.8, t=$ $2.095, p=.365)$. There was a significant interaction between $\mathrm{WMC}$ and $\mathrm{WM}$ load $(\beta=122.88, t$ $=2.046, p=.0411 ;$ Figure $1 \mathrm{~A})$ : The effects of WMC on the $\mu$ component of participants' $\mathrm{RT}$ distributions were stronger in the medium load condition $(\beta=-111.9[-195.0,-28.2])$ than in the high $(\beta=-53.9[-137.2,29.6])$ and low load conditions $(\beta=11.3[-72.1,94.7])$. There was also a significant interaction between WMC and task $(\beta=-114.805, t=2.341, p=.0195$; Figure 1B): When participants had just judged liking, the higher their WMC, the smaller the $\mu$ component of their RT distributions ( $\beta=-108.77[-181.1,-36.4])$. There was no effect of WMC when they had just judged beauty $(\beta=6.03[-57.5,69.6])$. No other main effect or interaction was significant (all $p \mathrm{~s}>.139)$. 


\section{Figure 1}

$\mu$ Component of Participants' Response Time Distributions in the WM Task
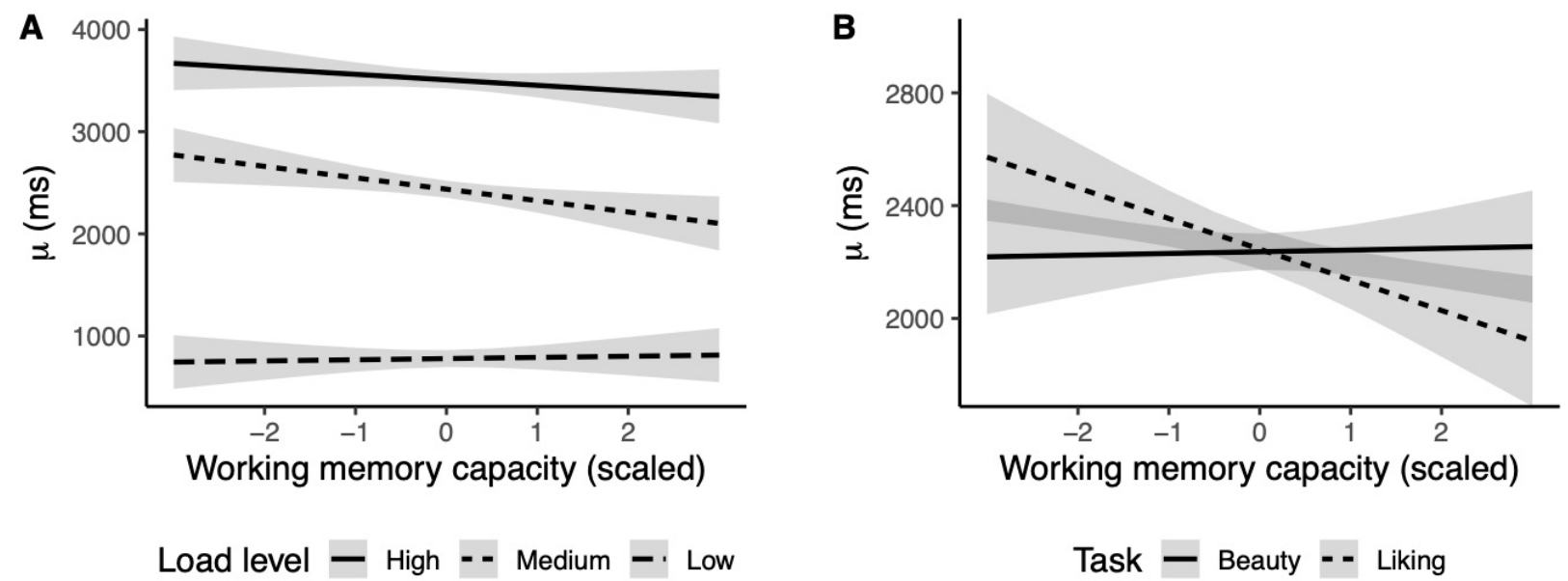

Note. A: $\mu$ component of participants' response time distributions in the WM task as a function of WM load condition.

B: $\mu$ component of participants' response time distributions as a function of task condition

For the $\sigma$ component, the intercept was $\beta_{0}=273.48 \mathrm{~ms}(t=19.271, p<.0001)$. The $\sigma$ component was greater when WM load was high $(414.4 \mathrm{~ms}[366,463])$ than when it was medium (308.7 ms [260, 357]; $\beta=-105.75, t=3.042, p=.0024$, Holm-Bonferroni corrected), and greater when it was medium than when it was low $(97.3 \mathrm{~ms}[49,146] ; \beta=-211.38, t=$ $6.081, p<.0001$, Holm-Bonferroni corrected). The effect of WMC on the $\sigma$ component was significant $(\beta=-45.21, t=3.175, p=.0016)$ : The greater participants' WMC, the smaller the $\sigma$ component of their RT distributions. However, this main effect was qualified by a two-way interaction between WM load and WMC $(\beta=-45.21, t=3.175, p=.0016)$. The effect of WMC on the $\sigma$ component was stronger in the medium load condition $(-83.3[-131.7,-34.9])$ than in the 
low load condition $(1.59[-46.8,50.0])$, but not than in the high load condition $(-53.9[-102.4,-$ 5.5]). In turn, this two-way interaction was qualified by a three-way interaction (Figure 2) among memory load, WMC, and task: in the medium load condition WMC had an effect when participants rated liking $(-153.8[-226.6,-81.0])$ but not when they rated beauty $(-12.8[-76.7$, 51.1]). No other main effect or interaction was significant (all $p \mathrm{~s}>.074$ ).

\section{Figure 2}

$\sigma$ Component of Participants' Response Time Distributions in the WM Task

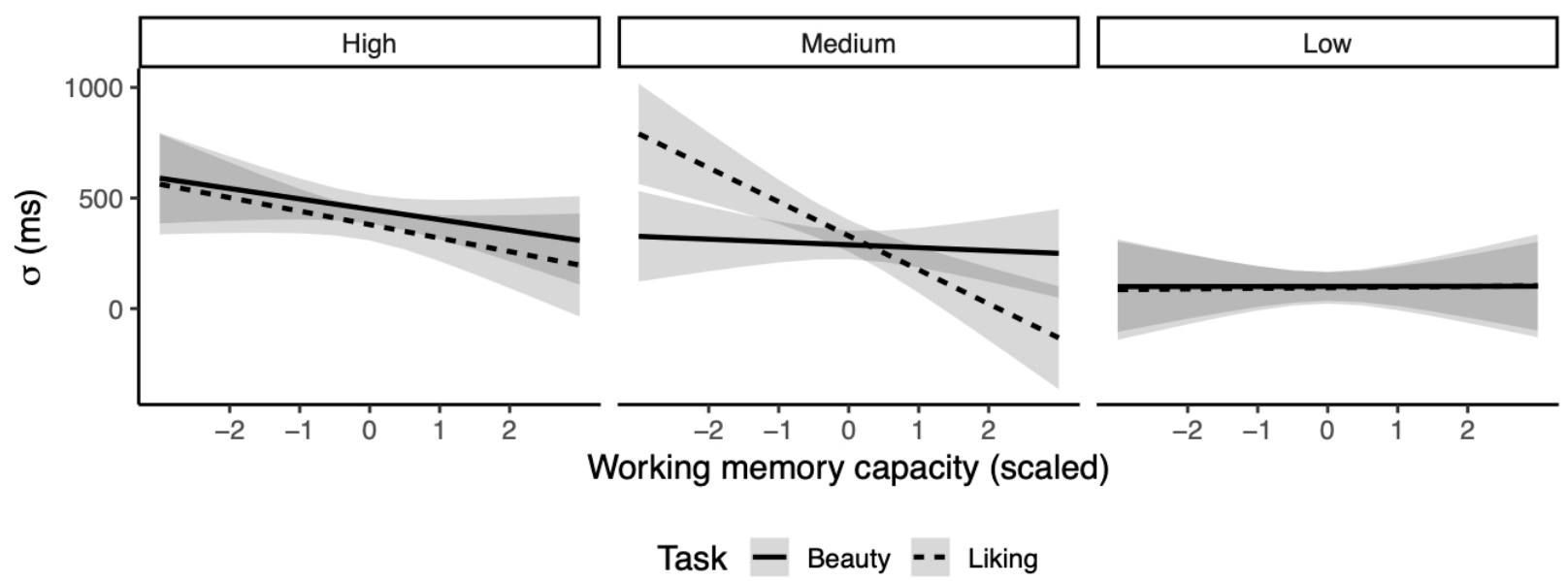

Note. $\sigma$ component of participants' response time distributions in the WM task as a function of WMC (horizontal axis), WM load (facets), and rating task (lines).

For the $\tau$ component, the intercept was $\beta_{0}=351 \mathrm{~ms}(t=18.717, p<.0001)$. WM load had a significant effect on $\tau(\beta=316.344, t=6.887, p<.0001)$ : It was greater in the high WM load condition $(510 \mathrm{~ms}[446,574])$ than in the low WM load condition $(113 \mathrm{~ms}[50,177])$, and greater in the medium WM load condition $(430 \mathrm{~ms}[366,494])$ than in the low WM load condition. None of the other main effects or interactions were significant (all $p \mathrm{~s}>.082$ ). 
In sum, visual WM load had a significant effect on the three RT parameters in the WM task. As load increased, response times became generally slower $(\mu)$, more heterogeneous $(\sigma)$, and included a greater preponderance of slow responses $(\tau)$. WMC contributed to performance, but in certain conditions only. Participants with greater WMC completed the matrices $(\mu)$ faster than participants with smaller WMC, but only in the medium load condition. Participants with greater WMC completed the matrices faster $(\mu)$ than participants with smaller WMC when they had just rated liking, but not when they had just rated beauty. Finally, participants with greater WMC were more consistent in their response times $(\sigma)$ than participants with smaller WMC in the medium load condition when they had just rated liking, but not when they had just rated beauty.

\section{Effects of Task, Memory Load, and WMC on Evaluative Judgments}

We had hypothesized that, if beauty and liking judgments make different demands on WM resources, we would find different effects of WM load on beauty and liking judgments, mediated by WMC. WM task had a significant effect on aesthetic ratings: Participants awarded higher ratings in the high WM load condition $(\mathrm{m}=3.25[3.03,3.46])$ than in the low memory load condition $(\mathrm{m}=3.09[2.89,3.30] ; \beta=0.153, t=2.803, p=.0035$, Holm-Bonferroni corrected), and higher ratings in the medium WM load condition $(\mathrm{m}=3.21[3.01,3.41])$ than in the low WM load condition $(\beta=-0.116, t=2.970, p=.0061$, Holm-Bonferroni corrected). No other main effect or interaction was significant (all $p \mathrm{~s}>.0824)$.

\section{Effects of Task, Memory Load, and WMC on Aesthetic Rating Time}


We had hypothesized that, if beauty and liking judgments make different demands on WM resources, we would find different effects of WM load on the time it took participants to make beauty and liking judgments, mediated by WMC. We first excluded trials with RTs under $150 \mathrm{~ms}$ and over the $3 \mathrm{rd}$ quartile +1.5 times the interquartile range $(331$ trials, $5.03 \%$ of the total). For the $\mu$ component the intercept was $\beta_{0}=1174.36 \mathrm{~ms}(t=33.744, p<.0001)$. Rating task had a significant $(\beta=153.40, t=2.204, p=.0283)$ : $\mu$ was greater when participants rated the beauty of the artworks $(1251 \mathrm{~ms}[1159,1343])$ than when they rated how much they liked them $(1098 \mathrm{~ms}[996,1199]$. No other main effect or interaction was significant (all $p \mathrm{~s}>.1017)$.

For the $\sigma$ component the intercept was $\beta_{0}=247.64 \mathrm{~ms}(t=13.418, p<.0001)$. No main effect or interaction was significant (all $p \mathrm{~s}>.0681)$.

For the $\tau$ parameter the intercept was $\beta_{0}=530.13 \mathrm{~ms}(t=15.199, p<.0001)$. There was a significant three-way interaction (Figure 3) between task, WM load, and WMC $(\beta=342.55, t=$ $1.998, p=.0466)$. Whereas participants' WMC had no effect on the $\tau$ parameter when they rated beauty or liking in the medium $\left(\beta_{\text {beauty }}=25.4[-131.9,182.8] ; \beta_{\text {liking }}=55.0[-124.3,234.2]\right)$ and high $\left(\beta_{\text {beauty }}=84.7[-72.6,242.1] ; \beta_{\text {liking }}=13.6[-165.7,192.8]\right) \mathrm{WM}$ load conditions, in the low WM condition, the higher participants' WMC, the lower their $\tau$ values when rating liking ( $\beta_{\text {liking }}$ $=-210.9[-390.1,-31.6])$, but not when rating beauty $\left(\beta_{\text {beauty }}=102.1[-55.3,259.5]\right)$. No other main effect or interaction was significant (all $p \mathrm{~s}>.093$ ). 


\section{Figure 3}

$\tau$ Component of Participants' Response Time Distributions in the Art Rating Task
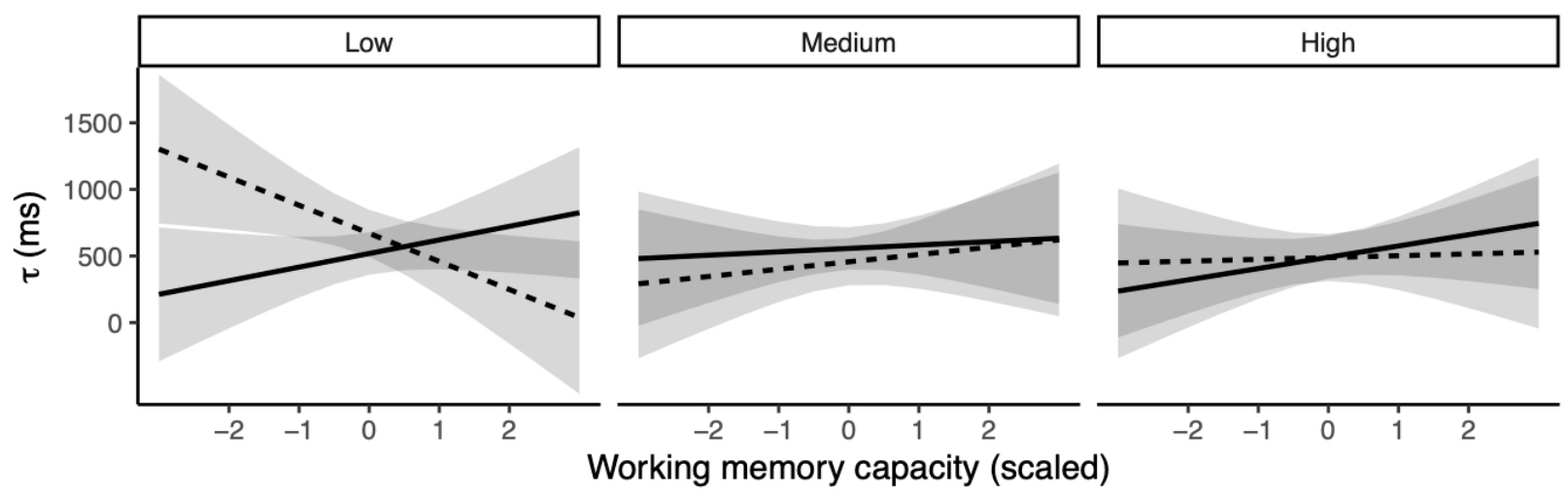

Task - Beauty -- Liking

Note. $\tau$ component of participants' response time distributions in the art rating task as a function of WMC (horizontal axis), WM load (facets), and rating task (lines).

In sum, while participants held information in memory about the location of the dots in the matrix they had to complete, it took them longer to rate beauty than to rate liking $(\mu)$. In the low load condition, participants with greater WMC had fewer slow responses $(\tau)$ than participants with smaller WMC when rating liking. However, there was no such advantage for performance when rating beauty.

\section{Discussion}

This study sought to determine how the evaluative judgments of beauty and liking differed in the way they engaged WM resources. We measured participants' WMC and asked 
them to perform two concurrent tasks: An evaluative judgment task (beauty or liking) and a short-term visuospatial memory task (with low, medium, and high load conditions).

As could be expected from the design of the WM task, as the load increased, participants made more mistakes on the WM task, and the advantage of higher WMC became clearer: In the low load condition, mistakes were independent of WMC, but in the high load condition, the greater participants' WMC, the fewer mistakes they made. WM load also influenced response times: As the load increased, response times became generally slower, more heterogeneous, and included more extremely slow responses. Participants with greater WMC completed the matrices faster than participants with smaller WMC, but only in the medium load condition. Taken together, this indicates that the low load condition was not too challenging for participants, regardless of their WMC: They all made few mistakes and completed the task consistently fast and with few slow responses. The medium load condition was more challenging for participants in general: They made more mistakes than in the low load condition, but the higher their WMC, the faster they could complete the task. The high load condition was most challenging of all, leading to more mistakes, and response times that were slower, more inconsistent and included more extremely slow responses. But although in the high WM load condition participants with higher WMC made fewer mistakes than those with lower WMC, they were not faster, indicative of a speed-accuracy tradeoff. Thus, in the medium load condition, higher WMC enabled speed, but in the high load condition higher WMC favored accuracy.

Results from the WM task supported our hypothesis that judgments of beauty and liking both engage WM resources, but that they do so in different ways. The greater participants' WMC, the faster they completed the WM task when they had just rated liking, but not when they had just rated beauty. Furthermore, in the medium load condition, the greater participants' 
WMC, the more consistent their response times when they had just rated liking, but not when they had just rated beauty. It seems, therefore, that participants with greater WMC were more effective than participants with lower WMC at controlling their attention with the goal of maintaining access to task-relevant information (the spatial arrangement of the 1,3, or 5 dots) when confronted with the competing task of judging liking. But when it came to judging beauty, regardless of WMC, participants found the task equally challenging.

The results of the evaluative judgment task also suggest that judgments of liking and beauty both make demands on WM resources, but in different ways. Regardless of whether participants were judging beauty or liking, the number of dots whose location participants had to store in an active state to be subsequently reproduced $(1,3$, or 5$)$ increased participants' ratings. This unexpected bias towards positive ratings under high WM load conditions was also reported by Mullennix and colleagues (2018) in their study of liking for abstract artworks. According to the variants of dual-process theory that ascribe affective processing to System 1 (e.g., Epstein, 1994), one of the consequences of increasing load is a greater likelihood that System 1 (which is affective) rather than System 2 will carry out processing. That increased affective cue might in turn be one of the mechanisms whereby ratings could increase for both liking and beauty. Although the outcome of liking and beauty judgments did not differ, the time it took participants to make those judgments did. While participants held information in memory about the location of the dots in the matrix they had to complete, it took them longer to rate beauty than to rate liking. Thus, participants' need to focus their attention on keeping the location of the dots in an active state to be able to subsequently complete the matrix delayed judgments of beauty to a greater extent than judgments of liking. In addition, in the low load condition, the greater 
participants' WMC, the fewer very slow responses when judging liking, but not when judging beauty. In the medium and high load conditions WMC was not an advantage.

In sum, our study provides evidence that judgments of beauty and liking draw on WM resources, but in different ways. The overall pattern of results suggests that the differences in the way and extent to which different evaluative judgments place demands on WM resources is much more nuanced than we originally expected. We did not find sweeping evidence that judging beauty requires more WM resources than judging liking (Brielmann \& Pelli, 2017; Mullennix et al., 2015; 2018; Leder et al., 2005). Rather, we found that both judgments rely on WM, but under certain circumstances, mainly defined by WM load and participants' WMC, judging beauty places more demands on participants' ability to control their attention with the goal of maintaining task-relevant information. We suggest that judging beauty requires attentional resources to keep goal-relevant information in an active state because it entails the additional process of matching objects' perceptual features to learnt beauty templates. This would explain why participants with greater WMC were able to carry out the WM task consistently faster when they had just judged liking but not when they had just judged beauty, and why when WM resources are taxed by the WM task, participants took longer to judge beauty than to judge liking. Further research is required to test this interpretation and, more generally, to clarify the role of executive functions in evaluative judgments, especially with regards to individual differences in WMC. 


\section{References}

Baayen, R. H., \& Milin, P. (2010). Analyzing reaction times. International Journal of Psychological Research, 3(2), 12-28.

Barr, D. J., Levy, R., Scheepers, C., \& Tily, H. J. (2013). Random effects structure for confirmatory hypothesis testing: Keep it maximal. Journal of memory and language, 68(3), 255-278. https://doi.org/10.1016/j.jml.2012.11.001

Berlyne, D. E. (1973). Interrelations of verbal and nonverbal measures used in experimental aesthetics. Scandinavian Journal of Psychology, 14(1), 177-184. https://doi.org/10.1111/j.1467-9450.1973.tb00107.x

Berridge, K. C., \& Kringelbach, M. L. (2015). Pleasure systems in the brain. Neuron, 86(3), 646664. https://doi.org/10.1016/j.neuron.2015.02.018

Brewer, G. A. (2011). Analyzing response time distributions. Methodological and theoretical suggestions for prospective memory researchers. Zeitschrift für Psychologie, 219, 117-124. https://doi.org/10.1027/2151-2604/a000056

Brielmann, A. A., \& Pelli, D. G. (2017). Beauty requires thought. Current Biology, 27(10), 15061513. https://doi.org/10.1016/j.cub.2017.04.018

Conway, A. R., Kane, M. J., Bunting, M. F., Hambrick, D. Z., Wilhelm, O., \& Engle, R. W. (2005). Working memory span tasks: A methodological review and user's guide. Psychonomic bulletin \& review, 12(5), 769-786. http://doi.org/10.3758/BF03196772

Cowan, N. (2017). The many faces of working memory and short-term storage. Psychonomic Bulletin \& Review, 24(4), 1158-1170. https://doi.org/10.3758/s13423-016-1191-6

Engle, R. W. (2018). Working Memory and Executive Attention: A Revisit. Perspectives on Psychological Science, 13(2), 190-193. https://doi.org/10.1177/1745691617720478 
Epstein, S. (1994). Integration of the cognitive and psychodynamic unconscious. American Psychologist, 49, 709-724. https://doi.org/10.1037/0003-066X.49.8.709

Heathcote, A., Popiel, S. J., \& Mewhort, D. J. K. (1991). Analysis of response time distributions: An example using the Stroop task. Psychological Bulletin, 109(2), 340-347. https://doi.org/10.1037/0033-2909.109.2.340

Ichikawa, S. (1983). Verbal memory span, visual memory span, and their correlations with cognitive tasks. Japanese Psychological Research, 25(4), 173-180. https://doi.org/10.4992/psycholres1954.25.173

Judd, C. M., Westfall, J., \& Kenny, D. A. (2017). Experiments with more than one random factor: Designs, analytic models, and statistical power. Annual Review of Psychology, 68(1), 601625. http://doi.org/10.1146/annurev-psych-122414-033702

Leder, H., Augustin, D., \& Belke, B. (2005). Art and cognition! Consequences for experimental aesthetics. Bulletin of Psychology and the Arts, 5, 11-20.

Lenth, R. (2018). emmeans: Estimated Marginal Means, aka Least-Squares Means. R package version 1.3.1. https://CRAN.R-project.org/package=emmeans

Marin, M. M., Lampatz, A., Wandl, M., \& Leder, H. (2016). Berlyne revisited: Evidence for the multifaceted nature of hedonic tone in the appreciation of paintings and music. Frontiers in Human Neuroscience, 10, 536. https://doi.org/10.3389/fnhum.2016.00536

Massidda, D. (2013). retimes: Reaction Time Analysis. R package version 0.1-2. https://CRAN.R-project.org/package=retimes

Miyake, A., Friedman, N. P., Rettinger, D. A., Shah, P., \& Hegarty, M. (2001). How are visuospatial working memory, executive functioning, and spatial abilities related? A latentvariable analysis. Journal of Experimental Psychology: General, 130(4), 621-640. 
https://doi.org/10.1037/0096-3445.130.4.621

Mullennix, J. W., Pilot, K. M., Steeves, T. A., \& Burns, J. C. (2018). The effects of cognitive load on judgments of titled visual art. Psychology of Aesthetics, Creativity, and the Arts, 12(2), 166-176. https://doi.org/10.1037/aca0000128

Mullennix, J. W., Wagner, N., Hetrick, B., Malloy, N., Jerome, R., \& Schminkey, M. (2015). Preference for visual art as a function of cognitive effort. North American Journal of Psychology, 17(3), 433-448.

R Core Team (2020). R: A language and environment for statistical computing. Version 4.0.2. R Foundation for Statistical Computing, Vienna, Austria. URL https://www.R-project.org/

Russell, P. A., \& George, D. A. (1990). Relationships between aesthetic response scales applied to paintings. Empirical Studies of the Arts, 8(1), 15-30. https://doi.org/10.2190/AU1R-6UXE-T14R-04WQ

Sherman, A., Grabowecky, M., \& Suzuki, S. (2015). In the working memory of the beholder: Art appreciation is enhanced when visual complexity is compatible with working memory. Journal of Experimental Psychology: Human Perception and Performance, 41(4), 898903. https://doi.org/10.1037/a0039314

Shipstead, Z., Harrison, T. L., \& Engle, R. W. (2015). Working memory capacity and the scope and control of attention. Attention, Perception, \& Psychophysics, 77(6), 1863-1880. https://doi.org/10.3758/s13414-015-0899-0

Singmann, H., Bolker, B., Westfall, J., \& Aust, F. (2015). afex: Analysis of factorial experiments. R package version 0.13-145. https://CRAN.R-project.org/package $=$ afex 